Pacific Journal of Mathematics

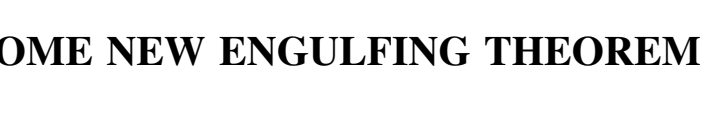




\title{
SOME NEW ENGULFING THEOREMS
}

\author{
FRED D. CRARY
}

\begin{abstract}
This paper extends and slightly improves existing piecewise linear radial engulfing theorems for compact polyhedra. For noncompact polyhedra, an engulfing theorem similar to the compact engulfing theorems of Stallings is proved. Two radial engulfing theorems for noncompact polyhedra similar to the compact radial engulfing theorems of Bing are proved.
\end{abstract}

The technique of engulfing has become one of the most useful tools in the study of topological embeddings. In this paper, we extend the current engulfing theorems, especially the radial engulfing theorems. We view engulfing from Stallings' viewpoint [10]: consider an open set $U$ in a manifold and determine conditions under which an isotopy of the manifold may be found such that the image of $U$ at the end of the isotopy contains a given polyhedron. We also wish to require that the isotopy be fixed on parts of the polyhedron that were initially contained in $U$. (Zeeman [14] presents a different viewpoint of engulfing.)

Radial engulfing, originally conceived by Connell [4], adds the restriction that the isotopy should only move in certain "preferred" directions. Connell's preferred directions were radial with respect to the origin in Euclidean space, hence the name "radial engulfing". Bing [1] generalized Connell's notion to other manifolds. Bing's radial engulfing theorem required that the dimension of the polyhedron to be engulfed be no greater than $m-4$ where $m$ is the dimension of the manifold (codimension 4). The main result of this paper (Theorem 2.1) is to prove Bing's theorem in codimension 3 and to slightly improve it in codimensions 4 and greater.

In Theorem 2.1 we require that the part of the polyhedron that is not initially engulfed be compact. In Theorem 3.3 we prove a (nonradial) engulfing theorem for noncompact polyhedra. Radial engulfing theorems for noncompact polyhedra are presented in Theorems 3.4 and 3.5 .

1. Notation and terminology. Our basic reference for terminology in the piecewise linear category (PL) is Hudson [7]. We use the terms "complex" (meaning "simplicial complex") and "polyhedron" interchangeably. The former will usually be used when we have a particular triangulation in mind. When not specified otherwise, all our complexes are built from closed simplexes. 
The $j$-skeleton of a $k$-complex $K$, written $K^{\prime}$, is the union of all simplexes of $K$ whose dimension is $j$ or smaller. If $L$ is a subcomplex of $K$, then the dual of $L$ in $K$ is the sub-complex of $K^{\prime}$ (the first barycentric subdivision of $K$ ) that is maximal with respect to the property of not intersecting $L$. If $L$ is a skeleton of $K$, then the dual of $L$ is also called the dual skeleton to $L$.

For approximating topological functions by PL ones, we use [7, Lemma 4.2] without explicit mention. We use the following definition of general position:

Definition. A map $f: K \rightarrow M$ from a complex $K$ to a PL $m$ manifold $M$ is said to be in general position if $f$ is a PL map, $f$ embeds every simplex of $K$, and for each collection $\sigma_{0}, \cdots, \sigma_{r}$ in $K, \operatorname{dim}(\cap$ $f\left(\right.$ int $\left.\left.\sigma_{t}\right)\right) \leqq \sum \operatorname{dim} \sigma_{t}-r m$.

If $f: K \rightarrow M$ is a map from a polyhedron $K$ to a PL manifold $M$ and $T$ is a triangulation of $K$, then $f$ is said to be in general position with respect to $T$ if the map $f: T \rightarrow M$ is in general position. A lemma of Hudson [7, Lemma 4.7] allows us to obtain general position approximations of PL maps. Both of these approximation lemmas allow us to keep the approximation maps $\epsilon$-close to the original maps, where $\epsilon$ is a continuous function into $(0, \infty)$.

Given an integer $p$, we say that a space $X$ is $p$-connected if for each integer $i \leqq p$, the homotopy group $\pi_{l}(X)=0$. The pair $(X, Y)$ of spaces, where $Y \subset X$, is said to be $p$-connected if for each $i \leqq p$, the relative homotopy group $\pi_{i}(X, Y)=0$.

Bing's substitute for a connectedness requirement for radial engulfing is as follows [1]. Suppose that $M$ is a PL $m$-manifold, $U$ is an open subset of $M$, and $\left\{A_{\alpha}\right\}$ is a collection of sets in $M$. Then we say that finite $k$-complexes in $M$ can be pulled into $U$ along $\left\{A_{\alpha}\right\}$ if given any finite $k$-complex $K$ in $M$ with $Q^{\prime}$ a subpolyhedron of $K$ that lies in $U$, then there exists a homotopy $H: K \times I \rightarrow M$ such that $H_{0}=I d, H_{1}(K) \subset U$, $H_{t}=I d$ on $Q^{\prime}$ and for each point $x \in K, H(x \times I)$ lies in an element of $\left\{A_{\alpha}\right\}$.

2. The compact case. In this section, we consider the case when the unengulfed portion of the polyhedron to be engulfed is compact. We consider the noncompact case in Section 3. Our main theorem is as follows.

THEOREM 2.1. Suppose that $M^{m}$ is a manifold without boundary, $U$ is an open subset of $M$,

$P$ is a closed polyhedron (not necessarily compact) in $M$ with $\operatorname{dim} P \leqq m-3$,

$Q$ is a subpolyhedron of $P$ with $Q \subset U$, 


\section{$R^{r}=\mathrm{Cl}(P-Q)$ is compact, and}

$\left\{A_{\alpha}\right\}$ is a collection of sets in $M$ such that finite $r$-complexes in $M$ can be pulled into $U$ along $\left\{A_{\alpha}\right\}$.

Then for each $\epsilon>0$, there is an engulfing isotopy $H: M \times I \rightarrow M$ and a compact set $E \subset M$ such that $H_{0}=I d, H_{t}=I d$ on $Q \cup(M-E)$, $H_{1}(U) \supset P$, and for each point $x \in M$ that is moved by $H$, there are $s$ elements of $\left\{A_{\alpha}\right\}$ such that $H(x \times I)$ lies in the $\epsilon$-neighborhood of the union of those $s$ elements where $s=1$ if $r=0, s=r$ if $0<r<m-3$, $s=r+1$ if $r=m-3$.

REMARKS. Theorem 2.1 extends and slightly improves Bing's radial engulfing theorem [1, Theorem A]. Bing's theorem was proved only for codimensions four and greater, and there his estimate of the track size was $r+1$ in all cases. In [11] and [12], P. Wright claimed Theorem 2.1 in the case $r=m-3$. He later withdrew this claim [13] and proved a codimension 3 radial engulfing theorem with somewhat worse bounds than those of Theorem 2.1. Radial engulfing theorems for codimension 3 have also been stated by R. D. Edwards and L. C. Glaser [6] and by C. Seebeck, III, [9]. Their estimates on the track size lie, in each case, between those of Theorem 2.1 and those of Wright [13].

Proof of Theorem 2.1. The isotopy we obtain is the result of a sequence of isotopies, each of which is the identity outside some $m$-cell in $M$. Thus we may take $E$ as the union of those cells and conclude that $H_{t}$ is the identity outside $E$. We will not mention $E$ again.

The proof is by induction on $r$, the dimension of the part of $P$ remaining to be engulfed. We divide the argument into three steps:

(1) $r=0$, or $r=1<m-3$,

(2) $1<r<m-3$, and

(3) $0<r=m-3$.

Proof of Step 1. $r=0$, or $r=1<m-3$. The hypothesis that finite $r$-complexes can be pulled into $U$ along $\left\{A_{\alpha}\right\}$ yields a homotopy $H^{1}: R \times I \rightarrow M$ as in the definition. Following Bing [1], we obtain successive approximations $H^{2}$ and $H^{3}$ to $H^{1}$, and a triangulation $T$ of $(R \times I) \cup(Q \times 0\}$ such that

$H_{0}^{3}=I d$,

$H^{3}[(R \times 1) \cup((Q \cap R) \times I] \subset U$,

each $H^{3}(x \times I)$ lies in an $\epsilon / 2$-neighborhood of some element of $\left\{A_{\alpha}\right\}$, $H^{3}$ is a PL homeomorphism on each simplex of $T$, and $H^{3}$ is in general position with respect to $T$. 
An upper bound on the dimension of the set of singularities of $H^{3}$ is $(r+1)+(m-3)-m=r-2$. For Step 1, we see that the set of singularities is empty, hence $H^{3}$ is an embedding.

To complete the proof of Step 1, we can invoke Bing's Engulfing Theorem B [1]. This consists of pushing "straight out" the $H^{3}$ images of vertical segments in $R \times I$. Each point that is moved generates a track near the image of one vertical segment and hence near a single element of $\left\{A_{\alpha}\right\}$. Since $s=1$ (for this step), we are finished with Step 1 .

Proof of Step 2. $1<r<m-3$. The proof of this step is the same as the induction step in Bing's proof of his Engulfing Theorem A [1], hence we omit all but the barest sketch. As in Step 1, we obtain a general position homotopy pulling $R$ into $U$. The singular set $X$ may have dimension as large as $r-2$. We form the simple shadow $L(X)$ of the singular set consisting of all the vertical segments in $R \times I$ containing points of $X$. Since $\operatorname{dim} L(X) \leqq r-1$, we may engulf the image of $L(X)$ by the induction hypothesis. Then the homotopy restricted to $(R \times I)-$ $L(X)$ shows how to pull the unengulfed part of $R$ into $U$ without any singularities; this part may then be engulfed as in Step 1.

Proof of Step 3. $0<r=m-3$. Again, by induction, we assume that the theorem holds for $k<r$. We construct $H^{1}, H^{2}$ and $T$ as in Step 1 except that each $H^{2}(x \times I)$ should lie in an $\epsilon / 8$-neighborhood of some element of $\left\{A_{\alpha}\right\}$ (instead of an $\epsilon / 4$-neighborhood). Let $T_{1}$ be a cylindrical subdivision of $T$ such that the image $H^{2}\left(\pi^{-1} \pi(\sigma)\right)$ of each cylinder in $T_{1}$ lies in the $\epsilon / 4$-neighborhood of some element of $\left\{A_{\alpha}\right\}$. Henceforth we will consider $R=R \times 0$ as a subcomplex of $T_{1}$ when viewing it as a complex. We extend $T_{1}$ to $Q=Q \times 0$.

We now construct $H^{3}$, a PL approximation of $H^{2}$, such that

$H_{0}^{3}=I d$,

$H^{3}[(R \times 1) \cup((Q \cap R) \times I)] \subset U$,

for $\sigma \in R, H^{3}(\sigma \times I)$ lies in the $\epsilon / 2$-neighborhood of some element of $\left\{A_{\alpha}\right\}$,

$H^{3}$ is a PL homeomorphism on each simplex of $T_{1}$, and

$H^{3}$ is in general position with respect to $T_{1}$.

Let $X$ be the set of singularities for $H^{3}$. In this case we have

$$
\operatorname{dim} X \leqq 2(r+1)-m=2(r+1)-(r+3)=r-1 .
$$

If we were to form the simple shadow $L(X)$ as in Step 2, we could have $\operatorname{dim} L(X)=r$ and would be unable to apply induction. The major part of what remains is finding a suitable substitute for $L(X)$ which has 
dimension $r-1$ or smaller. The method we use is based on Bing's shadow building techniques [1].

We start with a definition from Zeeman [14]. Let $K$ triangulate $L \times I$ and let $\pi: L \times I \rightarrow L$ be the natural projection. A simplex $\sigma \in K$ is called horizontal if $\pi \mid \sigma$ is a homeomorphism, and vertical if $\pi(\sigma)=$ $\pi(B d \sigma)$. Zeeman observed that, in a cylindrical triangulation or a subdivision of one, every simplex is either horizontal or vertical.

We next show that $X$ meets the walls of each top dimensional cylinder in $T_{1}$ in a sufficiently low dimensional set. More precisely, let

$$
X^{\prime}=X \cap\left[\left(R^{r-1} \times I\right) \cup(R \times\{0,1\})\right] .
$$

Since $X^{\prime}$ is contained in the singular set formed from intersections of the images of pairs of simplexes of $T_{1}$, at least one of which has dimension less than $r+1$, we have that

$$
\operatorname{dim} X^{\prime} \leqq(r+1)+r-m=r-2 .
$$

Thus the interiors of top dimensional simplexes of $X$ lie in the interiors of $(r+1)$-dimensional cylinders of $T_{1}$.

We next arrange matters so that all the $(r-1)$-simplexes of $X$ are horizontal. We may achieve this by subdividing so that $X$ is triangulated and then moving the vertices of $X$ so that no pair of them lies in the same vertical segment of $R \times I$. To simplify the notation, we suppose that $X$ already satisfies this property.

We now arrange matters so that the projection of the set of points of $X$ that lie on the same vertical segment as another point of $X$ (i.e., the projection of the singular set of $\pi \mid X)$ has dimension $r-2$ or smaller. We may do this by the techniques in Zeeman's Piping Lemma [14, Sublemma 1 of Lemma 48]. His technique is to observe that this condition is violated only if two or more $(r-1)$-simplexes of $X$ have the same projection. In that case, we pull these simplexes slightly to one side, each by a different amount, leaving their boundaries fixed. As before, we suppose that $X$ does not require this adjustment.

We now seek a special shadow $L^{\prime}$ for $H^{3}$. Before describing the desired properties of $L^{\prime}$, we define $X_{2}$ to be the set of singularities of $H^{3}$ arising from the intersection of the images of pairs of simplexes of $T_{1}$, at least one of which has dimension less than $r+1$; and $X_{3}$ to be the set of triple and higher order points of $H^{3}$. We then observe that $\operatorname{dim} X_{2} \leqq$ $r-2$, and $\operatorname{dim} X_{3} \leqq r-3$. Then we wish $L^{\prime}$ to satisfy the following properties:

$\operatorname{dim} L^{\prime} \leqq r-1$,

$L^{\prime}$ is the union of vertical segments of $R \times I$, 
$X_{2} \cup X_{3} \subset L^{\prime}$

if $y, z \in X$ with $H^{3}(y)=H^{3}(z)$, then $y \in L^{\prime}$

if and only if $z \in L^{\prime}$, and

if $y \in X-L^{\prime}$, then the vertical segment through

$y$ contains no other point of $X$.

Let $X_{4}$ be the set of points $x$ of $X$ such that the vertical segment through $x$ contains another point of $X$. Then let

$$
L^{\prime}=L\left(X_{2} \cup X_{3} \cup H^{3^{-1}} H^{3}\left(X_{4}\right)\right) \text {. }
$$

$L^{\prime}$ clearly satisfies all but the first property we desired. The first property follows since the interiors of $(r-1)$-simplexes of $X$ (in $\left.T_{2}\right)$ have disjoint projections into $R$.

Then $R \times I$ collapses cylinderwise to $L^{\prime}$ (for some cylindrical triangulation of $R \times I$ which has $L^{\prime}$ as a subcomplex). We begin our engulfing as before by engulfing $L^{\prime}$ by an engulfing isotopy $H: M \times$ $\left[0, \frac{1}{2}\right] \rightarrow M$ such that

$$
\begin{aligned}
& H_{0}=I d, \\
& H_{t}=I d \text { on } Q \cup H^{3}[(R \times 1) \cup((Q \cap R) \times I)], \\
& H_{\frac{1}{2}}(U) \supset H^{3}\left(L^{\prime}\right), \text { and }
\end{aligned}
$$

for each point $x \in M$, there are $r-1$ elements of

$\left\{A_{\alpha}\right\}$ such that $H\left(x \times\left[0, \frac{1}{2}\right]\right)$ lies in the

$\epsilon$-neighborhood of the union of these $r-1$ elements.

We note that if $r=1$, then $L^{\prime}=\varnothing$ and we may take $H_{t}=I d$ for $0 \leqq t \leqq \frac{1}{2}$.

Let $C\left(L^{\prime}\right)$ be a cylindrical neighborhood of $L^{\prime}$ such that $\mathrm{Cl}\left[H^{3}\left(C\left(L^{\prime}\right)\right)\right] \subset H_{\frac{1}{2}}(U)$. Then $R \times I$ collapses to $(R \times 1) \cup \overline{C\left(L^{\prime}\right)}$ and this collapse may be achieved by a cylinderwise collapse. $(R \times I)-$ $C\left(L^{\prime}\right)$ consists of a collection of vertical segments of $R \times I$. The image under $H^{3}$ of one of these segments intersects at most one other such image. Let $Y$ be the set whose elements are either the union of two such intersecting images or one such image that does not meet any other.

We extend $H$ to $\left[\frac{1}{2}, 1\right]$ by reversing the cylinderwise collapse mentioned in the previous paragraph. In "uncollapsing" each cylinder, we keep the track of each point close to the image of a vertical segment in that cylinder. Thus we may require that each track $H\left(x \times\left[\frac{1}{2}, 1\right]\right)$ lie in an $\epsilon / 2$-neighborhood of some element of $Y$. But each element of $Y$ lies in the $\epsilon / 2$-neighborhood of the union of some two elements of $\left\{A_{\alpha}\right\}$, hence each $H\left(x \times\left[\frac{1}{2}, 1\right]\right)$ lies in the $\epsilon$-neighborhood of the union of those two elements. Combining this with the estimate of track size on the interval $\left[0, \frac{1}{2}\right]$ completes the proof. 
Remarks. Zeeman's Piping Lemma techniques [14, Lemma 48] could have been used instead of Bing's shadow building techniques to prove Step 3. Such a proof yields the same estimates as the proof by shadow building (the details of such a proof are included in [5]). In fact, the two means of proof are actually different ways of looking at the same process - if we imagine pushing the image of the homotopy, in addition to the image of $U$, ahead of us when we pass through an unengulfed singularity the second time, we will have built pipes in just the same manner as Zeeman does.

Bing [2] shows how a map of $R \times I$ may be placed in "vertical general position"; that is, in general position so that its singularities are "nice" with respect to the projection map. Had this result $[2$, Theorem 2.1] been available to us, the construction of the shadow for Step 3 would have been greatly simplified.

3. The noncompact case. When considering the engulfing of noncompact (infinite) polyhedra, we are quickly led to the realization that connectedness is not the whole story and that we are limited to essentially finite techniques, at least at present. The following example seems to be the common one to illustrate the fact that connectivity is not enough.

EXAMPLE 3.1. Let $U$ be the open unit ball in $E^{n}=E^{1} \times E^{n-1}$. Let $Z$ be the product of the positive integers in the $E^{1}$ factor with the origin in the $E^{n-1}$ factor. Although $E^{n}$ is contractible, there is no way to engulf $Z$ with $U$ since, for example, $U$ is contained in a compact set, $\bar{U}$, but $Z$ is not.

To use the essentially finite techniques available, we need not only to have locally finite polyhedra as our target for engulfing, but also to require that infinitely many of its simplexes not "bunch up", but rather tend to the "ends" of the manifold. To make this notion precise we will define the concept of the end of a manifold. Very loosely, this is a "place" where the manifold becomes infinite (if indeed it does). In this sense, we would like to say that $E^{1}$ has two ends which we might call $+\infty$ and $-\infty$. The difficulty in defining an end lies in that we are defining something that is not there. We get around this by defining "how to go there from here".

Definition. An end $E$ of a manifold $M$ is a function from the collection of compact subsets of $M$ into the collection of nonempty open subsets of $M$ such that

$E(C)$ is a component of $M-C$, and

if $C_{1} \subset C_{2}$, then $E\left(C_{1}\right) \supset E\left(C_{2}\right)$. 
To put the concept of an end to use, the following trivial lemma is convenient.

LEMMA 3.2. Suppose that $\left\{C_{i}\right\}$ is a sequence of compact subsets of a manifold $M$ that satisfies $C_{i} \subset$ Int $C_{i+1}$, and $\cup C_{i}=M$. Let $E$ be an end of $M$ and define $U_{i}=E\left(C_{i}\right)$. Then the sequence $\left\{U_{i}\right\}$ is such that each $U_{i} \neq \varnothing, U_{1} \supset U_{2} \supset \cdots$, and each $U_{i}$ is a component of $M-C_{i}$.

REMARK. One may use sequences $\left\{U_{i}\right\}$ as in the conclusion of the lemma to obtain an equivalent definition of ends.

We next give definitions of the properties we will need for our (nonradial) infinite engulfing theorem.

Definitions. A neighborhood of an end $E$ is any subset of $M$ of the form $E(C)$. A subset of $M$ tends to an end if it intersects each neighborhood of that end.

A subset $X$ of $M$ is uniformly $k$-connected at the ends of $M$ if for each compact subset $C$ of $M$ there is a compact subset $K$ of $M$ such that for each end $E$ of $M$ and each integer $i \leqq k$, maps of $S^{i} \rightarrow X \cap E(K)$ are null homotopic in $X \cap E(C)$. If $X=M$, then we say that $M$ is uniformly $k$-connected at its ends.

REMARK. We could give definitions for uniform connectedness at some of the ends of a manifold by restricting our attention to only those ends in the subcollection with which we are concerned. Definitions of this sort will be alluded to in remarks on simple improvements of some of the theorems we will prove.

We are now ready to state and prove our (nonradial) infinite engulfing theorem. Other infinite engulfing theorems have been given in [3] and [8].

THEOREM 3.3. Suppose $M^{m}$ is an m-manifold,

$U$ is an open subset of $M$,

$P$ is a closed, locally finite polyhedron in Int $M$ with $\operatorname{dim} P \leqq m-3$,

$Q$ is a closed subpolyhedron of $P$ with $Q \subset U$,

$R^{r}=\mathrm{Cl}(P-Q)$,

if $P$ tends to an end $E$ of $M$, then so does $U$,

$(M, U)$ is $r$-connected,

$M$ is uniformly $r$-connected at its ends,

and $U$ is uniformly $(r-1)$-connected at the ends of $M$.

Then there exists an engulfing isotopy $H: M \times I \rightarrow M$ such that $H_{0}=I d, H_{t}=I d$ on $Q$, and $H_{1}(U) \supset P$.

Remark. It would be enough if $M$ and $U$ were uniformly connected at only those ends of $M$ that $R$ tends to. 
Proof of Theorem 3.3. Choose a sequence $\left\{C_{i}\right\}$ of compact subsets of $M$ such that $\cup C_{i}=M, C_{i} \subset \operatorname{Int} C_{i+1}$ for $i \geqq 1$, and for each end $E$ of $M$ and for each map $f: D^{r} \rightarrow E\left(C_{i+1}\right)$ with $f(\operatorname{Bd} D) \subset U$, there is a homotopy $\phi: D \times I \rightarrow M$ such that $\phi_{0}=f, \phi_{1}(D) \subset U, \phi_{t}=f$ on $\operatorname{Bd} D$, and $\phi(D \times I) \subset E\left(C_{i}\right)$.

The proof is by induction on $r$, thus we first suppose $r=0$ and find a homotopy $h: R \times I \rightarrow M$ such that $h_{0}=I d, h_{1}(R) \subset U, h(R \times I) \subset$ Int $M$, and for each $p \in R$, if $p \in E\left(C_{i+1}\right)$ for some end $E$ and some $i \geqq 1$, then $h(p \times I) \subset E\left(C_{i}\right)$. We extend $h$ to $Q=Q \times 0$ via the identity, approximate it by a PL nondegenerate map that agrees with $h$ on $P=P \times 0$, and then place it in general position (again so that the result agrees with $h$ on $P)$. After doing this, we find that the resulting map (which we continue to call $h)$ embeds $Q \cup(R \times I)$. Since $Q \cup(R \times I)$ is locally finite (because $P$ was), we may find for each point $p \in R$ an open subset $V_{p}$ of $M$ such that $V_{P} \supset h(p \times I), V_{P} \cap Q=\varnothing, V_{P} \cap V_{P^{\prime}}=\varnothing$ if $p \neq p^{\prime}$, and if $p \in E\left(C_{\imath+1}\right)$, then $V_{p} \subset E\left(C_{l}\right)$. We may then push along the $\operatorname{arc} h(p \times I)$ to engulf $p$ in the standard manner taking care to move no point outside $V_{p}$. Since the resulting isotopies have disjoint support, we may perform them simultaneously and then extend to all of $M$ via the identity to obtain the desired isotopy $H$.

Suppose now that $0<r<m-3$ and that the theorem has been proved for integers $k$ such that $0 \leqq k<r$. By induction we assume that the $(r-1)$-skeleton of $R$ is already contained in $U$ and, in fact, that it is a subset of $Q$. We construct a PL general position homotopy $h: R \times$ $I \rightarrow M$ such that $h_{0}=I d, h[(R \times 1) \cup((Q \cap R) \times I)] \subset U$,

$h(R \times I) \subset$ Int $M$, and

for each $\sigma^{r} \in R$, if $\sigma \subset E\left(C_{1+1}\right)$ for some end $E$ and integer $i \geqq 1$, then $h(\sigma \times I) \subset E\left(C_{l}\right)$.

Then, in the manner of the previous theorems, we construct a shadow $L$ for $h$ having dimension $<r$ and engulf its image under $h$ by induction. The remainder of $R \times I$ is embedded by $h$ and we engulf it by "uncollapsing" some cylindrical triangulation. For this sketch to go through, we need to establish the following facts. (1) $h(R \times I)$ is closed and locally finite, (2) $h(L)$ is closed, locally finite and has dimension $<r$, and (3) after engulfing $h(L)$, we are able to engulf the remaining (infinite) portion of $h(R \times I)$ by an isotopy. The restrictions we have placed on $h$ make these fairly easy to prove.

The last inductive case we have to consider is when $r=m-3$. In this case we meet the same problem that we met in Theorem 2.1 since the simple shadow may have dimension $r$ and thus block the induction. We cannot proceed exactly as in Step 3 of Theorem 2.1 due to the presence of 
unengulfed singularities after the inductive step. The difficulties can be solved by using refinements due to Bing (he used them to improve an engulfing lemma of Černavskiï, see [1, page 4]).

We follow the proof of the case where $r<m-3$ to the point of creating a PL general position homotopy $h: R \times I \rightarrow M$. We then depart from that proof by constructing a different shadow. Let $W$ be a cylindrical triangulation of $R \times I$. We may suppose that $h$ is a linear homeomorphism on each simplex of $W$, and if $\sigma_{i}, i=0,1, \cdots, n$, are open simplexes of $W$, then

$$
\operatorname{dim} \cap h\left(\sigma_{\imath}\right) \leqq\left(\sum \operatorname{dim} \sigma_{\imath}\right)-n m
$$

Let $X$ be the singular set of $h$, then $\operatorname{dim} X \leqq r-1$. Let $X_{1}=$ $X \cap h^{-1} h$ ( $r$-skeleton of $W$ ); then $\operatorname{dim} X_{1} \leqq r-2$. Let $X_{2}$ be the set of triple and higher order points of $h$; then $\operatorname{dim} X_{2} \leqq r-3$. We then seek a shadow $L$ with the following properties: $\operatorname{dim} L \leqq r-1, L$ is the union of vertical segments of $R \times I, L \supset X_{1} \cup X_{2}$, if $x^{\prime}, x^{\prime \prime} \in X$ with $h\left(x^{\prime}\right)=h\left(x^{\prime \prime}\right)$, then $x^{\prime} \in L$ if and only if $x^{\prime \prime} \in L$, and if $x \in X-L$, then the vertical segment through $x$ contains no other point of $X$. Define $X_{3}$ as the points $x$ of $X$ such that the vertical segment through $x$ meets $X$ in some point other than $x$. By pulling interiors of top dimensional simplexes of $X$ sideways as in Theorem 2.1 , we may assume that $\operatorname{dim} \pi\left(X_{3}\right) \leqq r-2$ where $\pi: R \times I \rightarrow R \times 0$ is projection. The shadow we seek is $L=$ $\pi^{-1} \pi\left(X_{1} \cup X_{2} \cup h^{-1} h\left(X_{3}\right)\right)$.

We begin our isotopy $H$ using the induction hypothesis to define $H: M \times\left[0, \frac{1}{2}\right] \rightarrow M$ as before so that $H_{\frac{1}{2}}(U) \supset Q \cup h(R \times 1) \cup h(L)$.

We will next engulf the remainder of $R$ by pushing down the images of the vertical segments of $R \times I$. To insure that no points are moved infinitely often and that the limit is continuous, we build some machinery. Choose a cylindrical subdivision $W^{\prime}$ of $W$ which has $X$ and $L$ as subcomplexes and let $\sigma_{1}, \sigma_{2}, \cdots$ be an enumeration of the simplexes of $(R \times 0)-L$. We require that this ordering be such that if $\sigma_{i}$ is a proper face of $\sigma_{l}$, then $i<j$. We call $\sigma_{i} \times I$ the column over $\sigma_{i}$ and let $\sigma_{i 1}, \sigma_{i 2}, \cdots, \sigma_{i k(t)}$ be the $\left(1+\operatorname{dim} \sigma_{i}\right)$-simplexes of the column over $\sigma_{i}$ ordered from the top (i.e., $R \times 1$ ) down. Our engulfing will push down these columns one at a time in the order of the $\sigma_{i}$.

Let us consider the vertical segments in $(R \times I)-L$. The image under $h$ of one of these segments intersects at most one other such image. Let $Y$ be the set whose elements are either (1) the union of two such intersecting images or (2) one such image that does not intersect any other. The elements of $Y$ form an upper semicontinuous decomposition of $h[(R \times I)-L]$, a separable metric space, and hence the decomposition space is metrizable. Using this decomposition, we construct a collection $G$ of open sets in $M-(Q \cup h(L))$ such that 
each element of $Y$ lies in an element of $G$,

if $y \in Y$ intersects an element of $G$, then $y$ lies in that element,

if $y \in Y, y \subset V \in G$ and $y \subset E\left(C_{i}\right)$ for some end $E$ of $M$ then $V \subset E\left(C_{i}\right)$,

if $V_{1}, V_{2} \in G$ and $V_{1} \cap V_{2} \neq \varnothing$, then $V_{1} \cap V_{2} \cap$ image $h \neq \varnothing$, and the elements of $G$ are "small with respect to the columns over the $\sigma_{i} "$.

To explain the last condition, we look at the way that the elements of $G$ intersect the images of the columns over the $\sigma_{i}$. If an element of $G$ intersects the image of the column over $\sigma_{i}$, then it must intersect the image of the column over each $\sigma_{j} \in \operatorname{star}\left(\sigma_{i}\right)$. If an element of $G$ contains an " $X$-shaped" element of $Y$ (that is, one that is the union of the images of two vertical segments), then it must intersect at least two such images of columns over stars. The last condition above then means that for each element $V$ of $G$ there is one such star so that $V$ meets $h(R \times I)$ only in the image of the column over that star unless $V$ contains an $X$-shaped element of $Y$ in which case two stars are permitted.

We can now define our isotopy as a sequence of pushes so that each point has a neighborhood that is moved only finitely many times and, if a point is moved at all, then its track lies in an element of $G$. To aid in this definition, we construct a sequence $G=G_{0}, G_{1}, \cdots$ of collections of open sets, each satisfying all the properties of $G$ given above, so that $G_{t+1}$ refines $G_{i}$ and the union of two intersecting members of $G_{i+1}$ lies in one element of $G_{i}$.

We now engulf successively the images $h$ (bottom face of $\sigma_{11}$ ), $h$ (bottom face of $\sigma_{12}$ ), etc. with pushes that always keep the image of the bottom face of $\sigma_{u j}$ covered after we passed $\sigma_{i j}$ in the ordering. We perform our pushes so that, if a point is moved by the $k$-th push, its track under that push lies in an element of $G_{k}$ that meets the $\sigma_{l l}$ associated with that push. The properties of the sequence $\left\{G_{i}\right\}$ insure that the track of each point moved by $H$ lies in some one element of $G$ and it is easy to check that no point is moved infinitely many times. This completes the proof of Theorem 3.3.

When trying to perform radial engulfing on noncompact polyhedra in the sense of Theorem 2.1, we must know that the structure of the manifold is such that parts of the open set $U$ "near the ends" of the manifold need not be pulled "far from the ends" in order to engulf a part of the polyhedron that is "near the end". We next introduce a property that will insure this.

Definition. Suppose that $U$ is an open subset of $M$ and $\left\{A_{\alpha}\right\}$ is a collection of subsets of $M$ such that finite $r$-complexes in $M$ can be pulled 
into $U$ along $\left\{A_{\alpha}\right\}$. Then we say that $\left\{A_{\alpha}\right\}$ uniformly preserves the ends of $M$ if for each compact subset $C$ of $M$ there is another compact subset $K$ such that, for each end $E$ of $M$, finite $r$-complexes in $E(K)$ can be pulled into $U$ along $\left\{A_{\alpha}\right\}$ by homotopies whose images lie in $E(C)$.

We next state and prove an infinite radial engulfing theorem.

THEOREM 3.4. Suppose that $M^{m}$ is an $m$-manifold,

$U$ is an open subset of $M$,

$P$ is a closed, locally finite polyhedron in Int $M$ with $\operatorname{dim} P \leqq m-3$,

$Q$ is a closed subpolyhedron of $P$ with $Q \subset U$,

$R^{r}=\mathrm{Cl}(P-Q)$, and

$\left\{A_{\alpha}\right\}$ is a collection of sets in $M$ such that

(1) finite $r$-complexes in $M$ can be pulled into $U$ along $\left\{A_{\alpha}\right\}$, and

(2) $\left\{A_{\alpha}\right\}$ uniformly preserves the ends of $M$.

Then for each continuous function $\epsilon: M \rightarrow(0, \infty)$, there exists an engulfing isotopy $H: M \times I \rightarrow M$ such that

$H_{0}=I d$,

$H_{t}=I d$ on $Q$,

$H_{1}(U) \supset P$, and

for each point $x \in M$ that is moved by $H$, there are

$s$ elements of $\left\{A_{\alpha}\right\}$ such that each point $p \in H(x \times I)$

lies in the $\epsilon(p)$-neighborhood of the union of these $s$ elements where

$$
s=\left\{\begin{array}{lll}
1 & \text { if } & r=0 \\
2^{r+1}-1 & \text { if } & 0 \leqq r<m-3 \\
2^{r+1} & \text { if } & 0<r=m-3 .
\end{array}\right.
$$

REMARK. It would be sufficient to assume that $\left\{A_{\alpha}\right\}$ uniformly preserves those ends of $M$ to which $R$ tends.

Proof of Theorem 3.4. Since $\left\{A_{\alpha}\right\}$ uniformly preserves the ends of $M$, we may choose a sequence $\left\{C_{l}\right\}$ of compact subsets of $M$ such that $\cup C_{1}=M, C_{1} \subset$ Int $C_{1+1}$, and for each end $E$ of $M$, each finite $r$-complex lying in $E\left(C_{i+1}\right)$ can be pulled into $U$ along $\left\{A_{\alpha}\right\}$ by a homotopy whose image lies in $E\left(C_{\imath}\right)$. The proof of Theorem 3.4 is by induction on $r$.

Case 1. $r=0$. We choose a homotopy $h: R \times I \rightarrow M$ such that $h_{0}=I d, h_{1}(R) \subset U$, for each point $x \in R, h(x \times I)$ lies in an element of $\left\{A_{\alpha}\right\}$, and if $x \in R$ and $x \in E\left(C_{i+1}\right)$ for some end $E$ of $M$, then $h(x \times I) \subset E\left(C_{i}\right)$. (Note that $Q$ and $R$ are disjoint when $r=0$ ). Having found $h$, we proceed as in the proof of Theorem 3.3 (in the case $r=0)$. However we must keep the $\epsilon(p)$ restriction in mind while 
making approximations and in the actual engulfing. In the case $r=0$, this poses no great problem, but we introduce some machinery that will aid in subsequent cases. This machinery allows us to regard $\epsilon$ as a constant function for most purposes of the proof.

We define the location $l(\sigma)$ of a simplex (point in this case) $\sigma$ of $R$ as $C_{i}$ where $i$ is the smallest integer such that $h(\sigma \times I) \subset$ Int $C_{i}$. Then let

$$
\delta(\sigma)=\min \{\epsilon(x): x \in l(\sigma)\} .
$$

We now proceed as in Theorem 3.3, however we make our approximations to $h$ as in Theorem 2.1 using $\delta(\sigma)$ instead of the constant $\epsilon$. In addition, we require that all approximations to $h \mid \sigma \times I$ lie in Int $l(\sigma)$ and that the isotopy engulfing $\sigma$ move no point outside $l(\sigma)$. The details are covered in the proofs of Theorems 2.1 and 3.3.

Case 2. $0<r<m-3$. We suppose that the theorem has already been proved for dimensions smaller than $r$. Then there is an engulfing isotopy $\Phi: M \times I \rightarrow M$ such that $\Phi_{0}=I d, \Phi_{t}=I d$ on $Q, \Phi_{1}(U) \supset R^{r-1}$ (the $(r-1)$-skeleton of $R$ ), and for each point $x \in M$, there are $2^{r}-1$ elements of $\left\{A_{\alpha}\right\}$ such that each point $p \in \Phi(x \times I)$ lies in the $\epsilon(p)$ neighborhood of the union of those elements of $\left\{A_{\alpha}\right\}$. We let $P_{1}=$ $\Phi_{1}^{-1}(P), Q_{1}=Q \cup \Phi_{1}^{-1}\left(R^{r-1}\right)$, and $R_{1}=\Phi_{1}^{-1}(R) . \quad R_{1}$ consists of images $\Sigma=\Phi_{1}^{-1}(\sigma)$ of $r$-simplexes $\sigma$ of $R$, with their boundaries contained in $Q_{1}$. For notational purposes, we will often regard $R_{1}$ as a "complex" with "simplexes" $\Sigma$.

By hypothesis, there is a homotopy $h: R_{1} \times I \rightarrow M$ such that $h_{0}=I d$, $h_{t}=I d$ on $Q_{1}, h_{1}\left(R_{1}\right) \subset U$, for each point $x \in R_{1}, h(x \times I)$ lies in an element of $\left\{A_{\alpha}\right\}$, and if $\Sigma \subset E\left(C_{i+1}\right)$, then $h(\Sigma \times I) \subset E\left(C_{i}\right)$. For each $\Sigma \in R_{1}$, we define $l(\Sigma)$ and $\delta(\Sigma)$ as in Case 1 using this homotopy $h$.

Next we obtain a PL general position approximation $h^{\prime}:\left(R_{1} \times I\right) \cup$ $\left(Q_{1} \times 0\right) \rightarrow M \quad$ satisfying: $\quad h_{0}^{\prime}=I d, \quad h^{\prime}\left[\left(R_{1} \times 1\right) \cup\left(\left(Q_{1} \cap R_{1}\right) \times I\right)\right] \subset U$, $h^{\prime}\left(R_{1} \times I\right) \subset$ Int $M$, for each $x \in \Sigma, \quad h^{\prime}(x \times I)$ lies in the $\frac{1}{2} \delta(\Sigma)$ neighborhood of some element of $\left\{A_{\alpha}\right\}, h^{\prime}(\Sigma \times I) \subset \operatorname{Int} l(\Sigma)$, and if $h(\Sigma \times I) \subset E\left(C_{i}\right)$, then $h^{\prime}(\Sigma \times I) \subset E\left(C_{l}\right)$. If $X$ is the singular set of $h^{\prime}$, we find that $\operatorname{dim} X \leqq r-2$ and for the simple shadow $L(X), \operatorname{dim} L(X) \leqq$ $r-1$. By the arguments given in the proof of Theorem 3.3, $L(X) \cup Q_{1}$ satisfies the hypotheses of this theorem. Therefore, by our induction hypothesis, we may find an engulfing isotopy $H: M \times\left[0, \frac{1}{4}\right] \rightarrow M$ such that $H_{0}=I d, H_{t}=I d$ on $Q_{1}, H_{4}(U) \supset Q_{1} \cup L(X)$, and for each point $x \in M$, there are $2^{r}-1$ elements of $\left\{A_{\alpha}\right\}$ such that each point $p \in H\left(x \times\left[0, \frac{1}{4}\right]\right)$ lies in the $\epsilon(p)$-neighborhood of the union of those elements of $\left\{A_{\alpha}\right\}$.

Then for each $\Sigma, h^{\prime}$ embeds $(\Sigma \times I)-L(X)$. By moving points close to elements of $\left\{h^{\prime}(x \times I): x \in \Sigma\right\}$, where "close" means "in a $\frac{1}{2} \delta(\Sigma)$-neighborhood", we may extend $H$ to $M \times\left[\frac{1}{4}, \frac{1}{2}\right]$ so that $H_{t}=I d$ on 
$Q_{1}, H_{\frac{1}{2}}(U) \supset P_{1}$, and for each $x \in M$, there is an element of $\left\{A_{\alpha}\right\}$ such that each point $p \in H\left(x \times\left[\frac{1}{4}, \frac{1}{2}\right]\right)$ lies in the $\epsilon(p)$-neighborhood of that element. Lastly, we define $H$ on $M \times\left[\frac{1}{2}, 1\right]$ by $H_{t}=$ $\Phi_{2 t-1} H_{\frac{1}{2}}\left(\frac{1}{2} \leqq t \leqq 1\right)$. Then $H_{t}$ satisfies the following for $\frac{1}{2} \leqq t \leqq 1$ :

$$
\begin{aligned}
& H_{t}=I d \text { on } Q, \\
& H_{1}(U) \supset P, \text { and }
\end{aligned}
$$

for each $x \in M$, there are $2^{r}-1$ elements of $\left\{A_{\alpha}\right\}$ such that each point $p \in H\left(x \times\left[\frac{1}{2}, 1\right]\right)$ lies in the $\epsilon(p)$-neighborhood of the union of those elements.

It remains only to check that this $H$ satisfies the conclusions of the theorem. The first three conditions are easily checked. To estimate the track sizes, we see that the maximum estimate will come from a point moved by all three parts of the isotopy. Summing the estimates for the three parts yields the required estimate.

Case 3. $0<r=m-3$. As in previous theorems, this case is proved similarly to Case 2 , except for the excessive dimension of the simple shadow. We engulf the $(r-1)$-skeleton of $R$, define $P_{1}, Q_{1}$ and $R_{1}$, and obtain a homotopy $h$ pulling $R_{1}$ into $U$ as before. We modify our definition of $\delta(\Sigma)$ in this case so that $\delta(\Sigma)=\min \left\{\epsilon(x): x \in C_{\imath+1}\right\}$ where $l(\Sigma)=C_{l}$. We do this because we anticipate that we may venture into $C_{\imath+1}-C_{l}$ during the engulfing of $\Sigma$ and thus need to have $\delta(\Sigma)$ reflect $\epsilon$ there. We continue as in Case 2 to obtain a PL general position homotopy $h^{\prime}$ with $h^{\prime} \mid \Sigma \times I \delta(\Sigma)$-close to $h \mid \Sigma \times I$ and construct a special shadow as in Theorem 3.3. We engulf the shadow keeping tracks $\epsilon$-close to $2^{r}-1$ elements of $\left\{A_{\alpha}\right\}$. When we engulf the rest of $R_{1}$, however, we stay $\epsilon$-close to 2 elements of $\left\{A_{\alpha}\right\}$ instead of 1 element as in Case 2. Finally we extend $H$ to engulf $R$ by applying the initial isotopy. This last isotopy stays $\epsilon$-close to $2^{r-1}$ elements of $\left\{A_{\alpha}\right\}$. Adding these numbers yields the estimate of track size required.

REMARK. The track size estimates are so large in Theorem 3.4 because our hypotheses only concern finite polyhedra. This leads to a problem in piecing together the finite subpolyhedra into which we must divide the infinite polyhedron $R$. With hypotheses that yield "nice" homotopies of infinite polyhedra, we can reduce the track size estimates to those of Theorem 2.1.

Definition. Suppose that $U$ is an open subset of $M$ and $\left\{A_{\alpha}\right\}$ is a collection of subsets of $M$ such that locally finite $r$-complexes in $M$ can be pulled into $U$ along $\left\{A_{\alpha}\right\}$ (we define this by inserting the word 
"locally" in the appropriate places of the finite definition). Then we say that $\left\{A_{\alpha}\right\}$ strongly preserves the ends of $M$ if for each compact subset $C$ of $M$ and each locally finite polyhedron $P$ of dimension $\leq r$ in $M$, there is another compact subset $K$ of $M$ and a homotopy $h: P \times I \rightarrow M$ pulling $P$ into $U$ along $\left\{A_{\alpha}\right\}$ such that for each end $E$ of $M$ and each simplex $\sigma$ of $P$ with $\sigma \subset E(K)$ we have that $h(\sigma \times I) \subset E(C)$.

REMARK. The preceding definition is equivalent to requiring that there exist a proper homotopy pulling $P$ into $U$ along $\left\{A_{\alpha}\right\}$. Recall that a map is proper if inverse images of compact sets are compact.

THeOREM 3.5. Suppose that $M^{m}$ is an $m$-manifold,

$U$ is an open subset of $M$,

$P$ is a closed, locally finite polyhedron in Int $M$ with $\operatorname{dim} P \leqq m-3$,

$Q$ is a closed subpolyhedron of $P$ with $Q \subset U$,

$R^{r}=\mathrm{Cl}(P-Q)$, and

$\left\{A_{\alpha}\right\}$ is a collection of sets in $M$ such that

(1) locally finite $r$-complexes in $M$ can be pulled into $U$ along $\left\{A_{\alpha}\right\}$, and

(2) $\left\{A_{\alpha}\right\}$ strongly preserves the ends of $M$.

Then for each continuous function $\epsilon: M \rightarrow(0, \infty)$, there exists an engulfing isotopy $H: M \times I \rightarrow M$ such that $H_{0}=I d, H_{t}=I d$ on $Q, H_{1}(U) \supset P$, and for each point $x \in M$ that is moved by $H$, there are selements of $\left\{A_{\alpha}\right\}$ such that each point $p \in H(x \times I)$ lies in the $\epsilon(p)$-neighborhood of the union of those $s$ elements where $s=1$ if $r=0, s=r$ if $0<r<m-3, s=r+1$ if $r=m-3$.

The proof of Theorem 3.5 is almost identical to that of Theorem 2.1 with the precautions taken in Theorem 3.3. Hence we omit the proof.

4. Questions. Since Theorem 2.1 represents an improvement and extension of previous radial engulfing theorems, the first question that comes to mind is the following:

Question 4.1. Can the track size estimates in Theorem 2.1 be reduced? How about Theorem 3.4? Theorem 3.5?

In particular, it would be pleasing to see Theorem 3.4 with track sizes that are linear (or better) in the dimension rather than exponential.

The next question is concerned with the existence (or nonexistence) of "infinite" techniques for handling singularities instead of the essentially finite ones we have used.

Question 4.2. Under what conditions can we engulf infinite polyhedra which are not locally finite? 
Question 4.3. Under what conditions can we engulf polyhedra that are not closed in $M$ ?

Here, the limit points of the polyhedron that are not in the polyhedron could cause difficulties.

\section{REFERENCES}

1. R. H. Bing, Radial engulfing, Conference on the Topology of Manifolds, 1967 (John G. Hocking, ed.), 1-18, Prindle, Weber and Schmidt, Boston, 1968.

2. - Vertical general position, to appear in the Proceedings of the 1974 Utah Topology Conference, Springer-Verlag.

3. A. V. Černavskiǐ, Isotopy of elements and spheres in $n$-dimensional space for $k<\frac{2}{3} n-1$, Dokl. Akad. Nauk SSSR 158 (1964), 62-65 or Soviet Math. Dokl., 5 (1964), 1194-1197.

4. E. H. Connell, Approximating stable homeomorphisms by piecewise linear ones, Ann. of Math., 78 (1963), 326-338.

5. F. D. Crary, Some new engulfing theorems, Ph.D. Thesis, The University of Wisconsin-Madison, August, 1973. Also Mathematics Research Center Technical Summary Report \# 1365, October, 1973.

6. R. D. Edwards and L. C. Glaser, A method for shrinking decompositions of certain manifolds, Trans. Amer. Math. Soc., 165 (1972), 45-56.

7. J. P. F. Hudson, Piecewise Linear Topology, Mathematics Lecture Notes Series, W. A. Benjamin, New York, 1969.

8. T. B. Rushing, Infinite engulfing, preprint.

9. C. L. Seebeck, III, Collaring an $(n-1)$-manifold in an $n$-manifold, Trans. Amer. Math. Soc., 148 (1970), 63-68.

10. J. R. Stallings, The Piecewise-linear structure of Euclidean space, Proc. Cambridge Philos. Soc., 58 (1962), 481-488.

11. P. Wright, A uniform generalized Schoenflies theorem, Bull. Amer. Math. Soc., 74 (1968), $718-721$.

12. __- A uniform generalized Schoenflies theorem, Ann. of Math., 89 (1969), 292-304.

13. _ Radial engulfing in codimension three, Duke Math. J., 39 (1971), 295-298.

14. E. C. Zeeman, Seminar on Combinatorial Topology (mimeographed notes), Chapter 7 , Engulfing, Institut des Hautes Etudes Scientifiques (1963).

Received August 22, 1974. This paper is a revision of the author's Ph.D. thesis. The author wishes to express his graditude to Prof. R. H. Bing for directing the thesis research. Sponsored by the United States Army under Contract No. DA-31-124-ARO-D-462.

Mathematics Research Center, The University of Wisconsin-Madison 



\section{Pacific Journal of Mathematics}

\section{Vol. 62, No. $1 \quad$ January, 1976}

Mieczyslaw Altman, Contractor directions, directional contractors and

directional contractions for solving equations . .................. 1

Michael Peter Anderson, Subgroups of finite index in profinite groups .........

Zvi Arad, Abelian and nilpotent subgroups of maximal order of groups of odd order

John David Baildon and Ruth Silverman, On starshaped sets and Helly-type theorems ..........................................

John W. Baker and R. C. Lacher, Some mappings which do not admit an

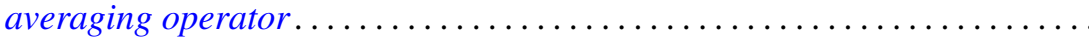

Joseph Barback, Composite numbers and prime regressive isols . . . . . . . . . .

David M. Boyd, Composition operators on $H^{p}(A) \ldots \ldots \ldots \ldots \ldots \ldots \ldots$

Maurice Chacron, Co-radical extension of PI rings . . . . . . . . . . . . .

Fred D. Crary, Some new engulfing theorems . . . . . . . . . . . . . . .

Victor Dannon and Dany Leviatan, A representation theorem for convolution transform with determining function in $L^{p} \ldots \ldots \ldots \ldots \ldots \ldots \ldots \ldots \ldots \ldots \ldots \ldots \ldots \ldots$

Mahlon M. Day, Lumpy subsets in left-amenable locally compact

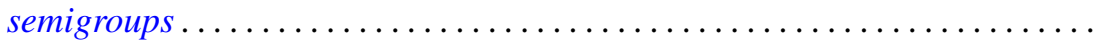

Michael A. Gauger, Some remarks on the center of the universal enveloping algebra of a classical simple Lie algebra . .

David K. Haley, Equational compactness and compact topologies in rings

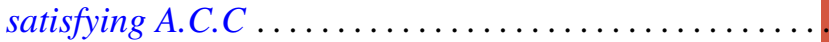

Raymond Heitmann, Generating ideals in Prüfer domains .

Gerald Norman Hile, Entire solutions of linear elliptic equations with

Laplacian principal part. .

Richard Oscar Hill, Moore-Postnikov towers for fibrations in which $\pi_{1}$ (fiber) is non-abelian

John Rast Hubbard, Approximation of compact homogeneous maps . .

Russell L. Merris, Relations among generalized matrix functions . .

V. S. Ramamurthi and Edgar Andrews Rutter, On cotorsion radicals ...

Ralph Tyrrell Rockafellar and Roger Jean-Baptiste Robert Wets, Stochastic

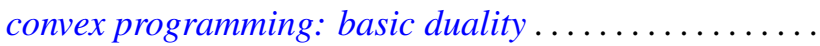

Alban J. Roques, Local evolution systems in general Banach spaces ..

I. Bert Russak, An indirect sufficiency proof for problems with bounded state variables.

Richard Alexander Sanerib, Jr., Ultrafilters and the basis property. .

H. A. Seid, The decomposition of multiplication operators on $L_{p}$-spaces . .

Franklin D. Tall, The density topology .................. 